\title{
Infectious Salpingitis
}

National Cancer Institute

\section{Source}

National Cancer Institute. Infectious Salpingitis. NCI Thesaurus. Code C78602.

An acute or chronic infectious process affecting the fallopian tube. Chlamydia

trachomatis, Neisseria gonorrhea, Mycoplasma, Staphylococcus, and Streptococcus are the most common causative agents. 